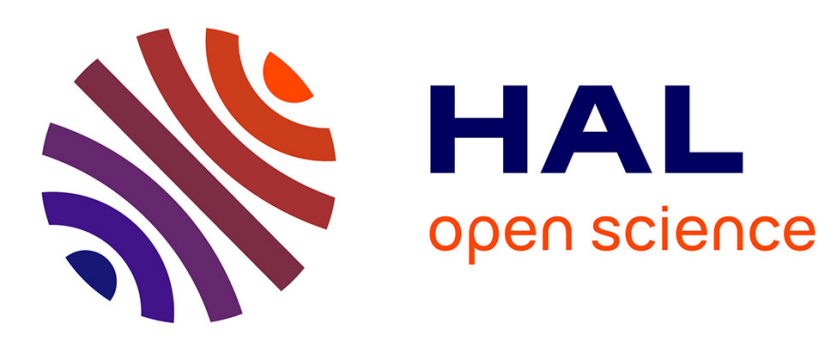

\title{
On the linear Boltzmann equation in evolutionary domains with absorbing boundary
}

Francesco Salvarani

\section{To cite this version:}

Francesco Salvarani. On the linear Boltzmann equation in evolutionary domains with absorbing boundary. 2013. hal-00803456v2

\section{HAL Id: hal-00803456 \\ https://hal.science/hal-00803456v2}

Preprint submitted on 15 Aug 2013

HAL is a multi-disciplinary open access archive for the deposit and dissemination of scientific research documents, whether they are published or not. The documents may come from teaching and research institutions in France or abroad, or from public or private research centers.
L'archive ouverte pluridisciplinaire HAL, est destinée au dépôt et à la diffusion de documents scientifiques de niveau recherche, publiés ou non, émanant des établissements d'enseignement et de recherche français ou étrangers, des laboratoires publics ou privés. 


\title{
ON THE LINEAR BOLTZMANN EQUATION IN EVOLUTIONARY DOMAINS WITH ABSORBING BOUNDARY
}

\author{
FRANCESCO SALVARANI
}

\begin{abstract}
We consider the linear Boltzmann equation in $\mathbb{R}^{d}, d \in \mathbb{N}$, under the effect of an absorbing moving barrier. We prove existence and uniqueness of the solution and consider the problem of the timeasymptotic convergence to equilibrium.

We then propose a numerical strategy to study the problem and provide quantitative results concerning some relevant test cases.
\end{abstract}

\section{INTRODUCTION}

This article aims to study some properties of the linear Boltzmann equation under the action of an absorbing moving surface.

This equation represents the time evolution of a population of point particles that interact with the medium trough absorption and scattering phenomena, by supposing that the mean free path between two consecutive interactions has the same order of magnitude as a macroscopic characteristic length.

The information on the particle system is given by the particle distribution density, a function that depends on space, time and, moreover, on some "internal" variables (i.e. variables that refer to a single particle of the system, typically the velocity of a single particle).

Many physical phenomena can be modelled by the linear Boltzmann equation. We quote, for example, the behaviour of a neutron population inside the core of a fission nuclear reactor [6] and the time evolution of the radiation, seen as a photons gas, inside the matter $[7,12]$.

However, the research on kinetic equations is mostly done in a static situation. The field of kinetic equations with moving elements - or in moving domains - is indeed still unexplored and, up to our knowledge, the only available results concern the Knudsen gas $[2,13,8]$.

Nevertheless, many reasons encourage the study of this kind of problems.

First of all, there is an intrinsic interest from a mathematical point of view, since a time-dependent absorbing barrier originates new phenomena that are absent when the domain is fixed, in particular when looking at the long-time evolution.

Moreover, a very practical argument suggests the research on the subject. Indeed, in general, the study of kinetic equations is very useful in establishing hierarchies of models, based on some underlying simple and physically

Key words and phrases. Linear Boltzmann equation, moving domains. 
relevant conservation laws, and obtained by an appropriate limiting procedure of the kinetic model when some scaling parameters tend to zero.

Therefore, this procedure allows to deduce very solid macroscopic models and it can be applied to many situations. We cite only, as example, the benefits of a kinetic deduction of models describing the fluid-structure interaction in hemodynamics, such as those studied in [11]. This approach is still unexplored, even when looking only to formal derivations.

In the kinetic description, the interaction of the particles with the medium is described by some functions, usually called "cross sections".

In this article we will consider quite general cross sections. However, for the sake of simplicity, we will avoid the presence of "holes" in the domain (which must be described by degenerate cross sections). We lead to $[4,3,5$, $10,9]$ the readers interested in understanding the peculiar features of this kind of cross sections.

This article is not exhaustive and it treats only some aspects of the problem under study. We will prove an existence and uniqueness theorem and then we will study the long time asymptotics of the problem. We note that our analysis of the long time behaviour is only a first step in understanding the rich behaviour of the system, and that further studies are necessary in order to obtain a deeper knowledge of the problem.

The paper is organized as follows: after the statement of the problem, given in the next section, we provide, in Sections 3 and 4, the theoretical background of the problem. Then, in Section 5, we explain the numerical method used for obtaining our numerical simulations, which will be presented and analysed in Section 6.

\section{THE PROBLEM}

Since the features of the absorbing moving barrier are the key aspect of the problem, we begin our analysis with a careful description of the requirements on the moving barrier itself that are needed for proving our results.

All the analysis will be performed in the framework of the $d$-dimensional Euclidean space $\mathbb{R}^{d}, d \in \mathbb{N}$.

Let $\psi: \mathbb{R}^{+} \times \mathbb{R}^{d} \rightarrow \mathbb{R}$ be a regular function, at least of class $C\left(\mathbb{R}^{+} ; C^{1}\left(\mathbb{R}^{d}\right)\right)$.

For all $t \geq 0$, we introduce the domain

$$
\Omega^{t}=\left\{x \in \mathbb{R}^{d}: \psi(t, x)<0\right\}
$$

and we define the absorbing moving barrier $\Gamma^{t}$ as the boundary of $\Omega^{t}$, i.e.

$$
\Gamma^{t}=\partial \Omega^{t} \text { for all } t \geq 0 .
$$

In what follows, we suppose some further basic properties on the family of domains $\Omega^{t}$, collected in the following definition:

Definition 2.1. The family of domains $\Omega^{t}, t \geq 0$, is said to be admissible if and only if:

$-\Omega^{t} \neq \emptyset$ for all $t \geq 0$;

- $\Omega^{t}$ is connected for all $t \geq 0$;

- the family $\Omega^{t}$ is uniformly contained in a fixed ball $B_{R}(0)$ of radius $R>0$ centred in the origin of the reference frame:

$$
\Omega^{t} \subset B_{R}(0) \text { for all } t \geq 0 \text {; }
$$


- for all $t \geq 0, \partial \Omega^{t}$ is without boundary.

Likewise, the moving barrier $\Gamma^{t}(t \geq 0)$ is admissible if and only if the corresponding family of domains $\Omega^{t}$ is admissible.

The paper considers the time evolution of a gas (or a set of identical point particles) described by a distribution function $f:=f(t, x, v)$. We will suppose that $x \in \mathbb{R}^{d}$ and that the velocities belong to a suitable velocity space $V \subseteq \mathbb{R}^{d}$. Possible examples of velocity spaces are the whole Euclidean space $\mathbb{R}^{d}$, the sphere $\mathbb{S}^{d-1}$ or the spherical shell $\mathbb{V}=\left\{v \in \mathbb{R}^{d}: 0<v_{m} \leq\right.$ $\left.|v| \leq v_{M}\right\}$.

The physical meaning of the distribution function is the following: if $\bar{X} \subset \mathbb{R}^{d}$ and $\bar{V} \subset V$, the integral

$$
\int_{\bar{X} \times \bar{V}} f(t, x, v) d x d v
$$

represents the number of gas molecules that, at time $t$, have position $x \in \bar{X}$ and velocity $v \in \bar{V}$.

We suppose moreover that the initial conditions have support contained in $\Omega^{0}$, and then that the particles interact with a fixed background.

The time evolution of $f$ is hence governed by the linear Boltzmann equation

$$
\frac{\partial f}{\partial t}+v \cdot \nabla_{x} f+a(t, x, v) f-K f=0, \quad(t, x, v) \in \mathbb{R}^{+} \times \mathbb{R}^{d} \times V,
$$

where

$$
K f(t, x, v):=\int_{V} k(t, x, v, w) f(t, x, w) d w .
$$

The problem is supplemented with the initial condition and with the constraint on the absorbing surface:

$$
f(0, x, v)=\left\{\left.\begin{array}{ll}
f^{\text {in }}(x, v) & \text { if }(x, v) \in \Omega^{0} \times V \\
0 & \text { otherwise }
\end{array} f(t, x, v)\right|_{x \in \Gamma^{t}}=0,\right.
$$

where $f^{\text {in }} \in L^{\infty}\left(\Omega^{0} \times V\right)$.

The interaction of the particles with the medium is described by the scattering operator $K$ and by the absorption term $a$.

The kernel $k$ of the scattering operator $K$ is a function of class $L^{\infty}\left(\mathbb{R}^{+} \times\right.$ $\left.\mathbb{R}^{d} \times V \times V\right)$, locally symmetric with respect to the velocity variables, i.e., for a.e. $(t, x) \in \mathbb{R}^{+} \times \mathbb{R}^{d}$

$$
k(t, x, v, w)=k(t, x, w, v) \text { for a.e. }(v, w) \in V \times V
$$

and such that

$$
0 \leq k(t, x, v, w) \text { for a.e. }(t, x, v, w) \in \mathbb{R}^{+} \times \mathbb{R}^{d} \times V \times V .
$$

Moreover we suppose that

$$
k(t, x, v, w)=0 \text { for a.e. } x \in \mathbb{R}^{d} \backslash \Omega^{t},
$$

for a.e. $(v, w) \in V \times V$, for all $t \in[0,+\infty)$, and that there exists a positive constant $M \in \mathbb{R}^{+}$such that

$$
M:=\sup _{(t, x, v) \in \mathbb{R}^{+} \times \mathbb{R}^{d} \times V} \int_{V} k(t, x, v, w) d w<+\infty .
$$


The absorption term $a$ is a function of class $L^{\infty}\left(\mathbb{R}^{+} \times \mathbb{R}^{d} \times V\right)$. We will suppose moreover that

$$
a \geq \bar{a}>0 \text { for a.e. } x \in \Omega^{t} \text { and } a=0 \text { for a.e. } x \in \mathbb{R}^{d} \backslash \Omega^{t},
$$

for a.e. $v \in V$ and for all $t \in[0,+\infty)$.

The functions $a$ and $k$ are the cross sections of our problem.

The main tool for studying Equation (2.1) is the backward absorbing time, defined as follows.

Definition 2.2. The backward absorbing time $\tau_{\Omega^{t}}(x, v)$ for a particle starting from $x \in \Omega^{t}$ in the direction $v \in V$, is defined as

$$
\tau_{\Omega^{t}}(x, v)=\inf \left\{\theta>0: x-\theta v \in \Gamma^{t-\theta}\right\} .
$$

If the set $\Theta:=\left\{\theta>0: x-\theta v \in \Gamma^{t-\theta}\right\}$ is empty, then $\tau_{\Omega^{t}}(x, v)=+\infty$.

\section{Existence And Uniqueness}

This section is devoted to the study of the basic properties concerning the well posedness of (2.1)-(2.2). However, before considering the main problem, we will need some results on the linear transport equation. We hence study the evolution equation governing the unknown $g$ given by

$$
\frac{\partial g}{\partial t}+v \cdot \nabla_{x} g+a(t, x, v) g=S(t, x, v), \quad(t, x, v) \in \mathbb{R}^{+} \times \mathbb{R}^{d} \times V .
$$

The absorption term $a \in L^{\infty}\left(\mathbb{R}^{+} \times \mathbb{R}^{d} \times V\right)$ is such that

$$
a \geq \bar{a}>0 \text { for a.e. } x \in \Omega^{t} \text { and } a=0 \text { for a.e. } x \in \mathbb{R}^{d} \backslash \Omega^{t}
$$

and the source term $S \in L^{\infty}\left(\mathbb{R}^{+} \times \mathbb{R}^{d} \times V\right)$ is such that

$$
S \geq 0 \text { for a.e. } x \in \Omega^{t} \text { and } S=0 \text { for a.e. } x \in \mathbb{R}^{d} \backslash \Omega^{t},
$$

for a.e. $v \in V$ and for all $t \in[0,+\infty)$.

The problem is supplemented with the initial condition and with the constraint on the absorbing barrier

$$
g(0, x, v)=\left\{\left.\begin{array}{ll}
g^{\text {in }}(x, v) & \text { if }(x, v) \in \Omega^{0} \times V \\
0 & \text { otherwise }
\end{array} g(t, x, v)\right|_{x \in \Gamma^{t}}=0\right.
$$

where $g^{\text {in }} \in L^{p}\left(\Omega^{0} \times V\right), 1 \leq p \leq+\infty$.

The method of characteristics gives the explicit form of the unique weak solution of the problem (3.1)-(3.2), namely

$$
\begin{aligned}
& g(t, x, v)=g^{\mathrm{in}}(x-t v, v) \exp \left(-\int_{0}^{t} a(s, x+(s-t) v, v) d s\right) \mathbb{1}_{\tau_{\Omega^{t}}(x, v)>t} \\
+ & \int_{\left(t-\tau_{\Omega^{t}}(x, v)\right)^{+}}^{t} \exp \left(-\int_{s}^{t} a(\tau, x+(\tau-t) v, v) d \tau\right) S(s, x+(s-t) v, v) d s .
\end{aligned}
$$

Under the hypotheses stated before, if $g^{\text {in }} \geq 0$ for a.e. $(x, v) \in \Omega^{0} \times V$, then $g \geq 0$ for a.e. $(t, x, v) \in \mathbb{R}^{+} \times \mathbb{R}^{d} \times V$. Moreover, $g=0$ for a.e. $x \in \mathbb{R}^{d} \backslash \Omega^{t}$, for a.e. $v \in V$ and for all $t>0$.

The main goal of the section is the proof of the following theorem: 
Theorem 3.1. Let $f^{\text {in }} \in L^{\infty}\left(\Omega^{0} \times V\right)$ be a non-negative function for a.e. $(x, v) \in \Omega^{0} \times V$. If the moving barrier $\Gamma^{t}, t \geq 0$, satisfies the requirements of Definition 2.1, then the initial-boundary value problem (2.1)-(2.2) admits a unique weak solution $f \in L^{\infty}\left(\left[0, t^{*}\right] \times \mathbb{R}^{d} \times V\right)$.

Moreover, $f \geq 0$ for a.e. $(t, x, v) \in\left[0, t^{*}\right] \times \mathbb{R}^{d} \times V, t^{*}>0$.

Proof. The strategy of proof is inspired by the classical representation of the solution in terms of the Dyson-Phillips expansion (see, for example, [1]).

We write the problem (2.1)-(2.2) in the integral form obtained by applying the method of characteristics. We obtain

$$
\begin{gathered}
f(t, x, v)=f^{\mathrm{in}}(x-t v, v) \exp \left(-\int_{0}^{t} a(s, x+(s-t) v, v) d s\right) \mathbb{1}_{\tau_{\Omega^{t}}(x, v)>t} \\
+\int_{\left(t-\tau_{\Omega^{t}}(x, v)\right)^{+}}^{t} \exp \left(-\int_{s}^{t} a(\tau, x+(\tau-t) v, v) d \tau\right) K f(s, x+(s-t) v, v) d s .
\end{gathered}
$$

The previous equation can be written in the equivalent form

$$
f=F\left(f^{\text {in }}\right)+T f
$$

where

$$
F\left(f^{\text {in }}\right)=f^{\text {in }}(x-t v, v) \exp \left(-\int_{0}^{t} a(s, x+(s-t) v, v) d s\right) \mathbb{1}_{\tau_{\Omega^{t}}(x, v)>t}
$$

and

$$
T h=\int_{\left(t-\tau_{\Omega^{t}}(x, v)\right)^{+}}^{t} \exp \left(-\int_{s}^{t} a(\tau, x+(\tau-t) v, v) d \tau\right) K h(s, x+(s-t) v, v) d s .
$$

We now prove that the series

$$
f=\sum_{n=0}^{+\infty} T^{n} F\left(f^{\mathrm{in}}\right)
$$

is a solution of Equation (3.4).

Let $t^{*} \in \mathbb{R}^{+}$. Since $f^{\text {in }} \in L^{\infty}\left(\Omega^{0} \times V\right), f^{\text {in }} \geq 0$ for a.e. $(x, v) \in \mathbb{R}^{d} \times V$ and $a(t, x, v) \geq 0$ for a.e. $(x, v) \in \mathbb{R}^{d} \times V$, then

$$
F\left(f^{\text {in }}\right)(t, x, v) \geq 0
$$

for a.e. $(t, x, v) \in\left[0, t^{*}\right] \times \mathbb{R}^{d} \times V$ and

$$
\left\|F\left(f^{\mathrm{in}}\right)\right\|_{L^{\infty}\left(\left[0, t^{*}\right] \times \mathbb{R}^{d} \times V\right)} \leq\left\|f^{\mathrm{in}}\right\|_{L^{\infty}\left(\Omega^{0} \times V\right)} .
$$

It is then easy to see that, for all $h \in L^{\infty}\left(\left[0, t^{*}\right] \times \mathbb{R}^{d} \times V\right)$

$$
\begin{gathered}
\left|T^{n} h(t, x, v)\right| \leq \int_{0}^{t}\left|K T^{n-1} h\right|(s, x+(s-t) v, v) d s \\
\leq M \int_{0}^{t}\left\|T^{n-1} h(s, \cdot, \cdot)\right\|_{L^{\infty}\left(\mathbb{R}^{d} \times V\right)} d s .
\end{gathered}
$$

By iteration on $n$, we finally deduce that

$$
\sup _{t \in\left[0, t^{*}\right]}\left\|T^{n} h(t, \cdot, \cdot)\right\|_{L^{\infty}\left(\mathbb{R}^{d} \times V\right)} \leq \sup _{t \in\left[0, t^{*}\right]} \frac{(M t)^{n}}{n !}\|h(t, \cdot, \cdot)\|_{L^{\infty}\left(\mathbb{R}^{d} \times V\right)} .
$$


The previous estimate proves that the linear application $T^{n}$ is continuous from the Banach space $L^{\infty}\left(\left[0, t^{*}\right] \times \mathbb{R}^{d} \times V\right)$ into itself, and that its norm verifies

$$
\left\|T^{n}\right\|_{\mathcal{L}\left(L^{\infty}\left(\left[0, t^{*}\right] \times \mathbb{R}^{d} \times V\right)\right)} \leq \frac{\left(M t^{*}\right)^{n}}{n !} .
$$

We can then conclude that each term of the series (3.5) is positive and that

$$
\left\|T^{n} F\left(f^{\text {in }}\right)\right\|_{L^{\infty}\left(\left[0, t^{*}\right] \times \mathbb{R}^{d} \times V\right)} \leq \frac{\left(M t^{*}\right)^{n}}{n !}\left\|f^{\text {in }}\right\|_{L^{\infty}\left(\Omega^{0} \times V\right)} .
$$

The space $L^{\infty}\left(\left[0, t^{*}\right] \times \mathbb{R}^{d} \times V\right)$ is a complete Banach space. Moreover, the series (3.5) is composed by positive terms and is normally convergent. Hence $f$, obtained as the limit of (3.5), exists and belongs to $L^{\infty}\left(\left[0, t^{*}\right] \times \mathbb{R}^{d} \times V\right)$.

Since the application $T^{n}$ is continuous on $L^{\infty}\left(\left[0, t^{*}\right] \times \mathbb{R}^{d} \times V\right)$, then

$$
\begin{aligned}
& f=\sum_{n=0}^{+\infty} T^{n} F\left(f^{\text {in }}\right)=F\left(f^{\text {in }}\right)+\sum_{n=1}^{+\infty} T^{n} F\left(f^{\text {in }}\right) \\
& =F\left(f^{\text {in }}\right)+T \sum_{n=0}^{+\infty} T^{n} F\left(f^{\text {in }}\right)=F\left(f^{\text {in }}\right)+T f .
\end{aligned}
$$

This shows that $f$ is solution of (3.4). The existence of a solution of class $L^{\infty}\left(\left[0, t^{*}\right] \times \mathbb{R}^{d} \times V\right)$ of the problem (2.1)-(2.2) is hence proved.

The uniqueness of the solution can be deduced as follows. Suppose that there exist two different solutions $f_{1}$ and $f_{2}$ of (3.4), which are of class $L^{\infty}\left(\left[0, t^{*}\right] \times \mathbb{R}^{d} \times V\right)$.

Hence, by linearity, the function $h=f_{1}-f_{2}$ (which is of class $L^{\infty}\left(\left[0, t^{*}\right] \times\right.$ $\left.\mathbb{R}^{d} \times V\right)$ ) satisfies the problem $h=T h$.

By iteration, $h=T^{n} h$ for all $n \in \mathbb{N}$. Hence,

$$
\begin{gathered}
\|h\|_{L^{\infty}\left(\left[0, t^{*}\right] \times \mathbb{R}^{d} \times V\right)} \leq\left\|T^{n}\right\|_{\mathcal{L}\left(L^{\infty}\left(\left[0, t^{*}\right] \times \mathbb{R}^{d} \times V\right)\right)}\|h\|_{L^{\infty}\left(\left[0, t^{*}\right] \times \mathbb{R}^{d} \times V\right)} \\
\leq \frac{\left(M t^{*}\right)^{n}}{n !}\|h\|_{L^{\infty}\left(\left[0, t^{*}\right] \times \mathbb{R}^{d} \times V\right)}
\end{gathered}
$$

for all $n \in \mathbb{N}$.

Hence $h=0$ for a.e. $(t, x, v) \in\left[0, t^{*}\right] \times \mathbb{R}^{d} \times V$, and the uniqueness of the solution in $L^{\infty}$ is proved.

Finally, since the series (3.5) is composed of positive terms, then the unique solution $f$ of (2.1)-(2.2) is positive.

\section{Convergence to equilibrium}

The condition $\Omega^{t} \subset B_{R}(0)$, for all $t \geq 0$, has an important consequence for the long-time asymptotics of the solution.

We will perform the computations for velocities belonging to the spherical shell $\mathbb{V}$ only. Straightforward modifications allow to treat the case $V=\mathbb{S}^{d-1}$.

In the absorption dominated case (i.e. when $\left.\bar{a} \geq\|k\|_{L^{\infty}\left(\left(0, t^{*}\right) \times B_{R}(0) \times \mathbb{V} \times \mathbb{V}\right)}\right)$, the following result holds:

Theorem 4.1. Let $f^{\text {in }} \geq 0$ for a.e. $(x, v) \in \Omega^{0} \times \mathbb{V}$, where

$$
\mathbb{V}=\left\{v \in \mathbb{R}^{d}: 0<v_{m} \leq|v| \leq v_{M}\right\} .
$$

If the absorbing barrier $\Gamma^{t}$ satisfies the prescriptions of Definition 2.1 and, moreover, $\bar{a} \geq\|k\|_{L^{\infty}\left(\left(0, t^{*}\right) \times B_{R}(0) \times \mathbb{V} \times \mathbb{V}\right)}$, then the unique solution $f$ of the 
initial-boundary value problem (2.1)-(2.2) asymptotically converges to zero with an explicit exponential rate for $t \rightarrow+\infty$. Moreover, the $L^{1}$-norm of the solution satisfies the following estimate:

$$
\begin{gathered}
\|p(t, \cdot, \cdot)\|_{L^{1}\left(B_{R}(0) \times \mathbb{V}\right)} \leq\left\|f^{\mathrm{in}}\right\|_{L^{1}\left(\Omega^{0} \times \mathbb{V}\right)} e^{-\left(\bar{a}-\|k\|_{L^{\infty}\left(\left(0, t^{*}\right) \times B_{R}(0) \times \mathbb{V} \times \mathbb{V}\right)}\right) t} \\
-e^{-\left(\bar{a}-\|k\|_{L^{\infty}}\right) t} \int_{0}^{t} \int_{\substack{\partial B_{R}(0) \times \mathbb{V} \\
v \cdot n_{x}>0}}\left|v \cdot n_{x}\right| f^{\text {in }}(x-\theta v, v) e^{-\|a\|_{L^{\infty}} \theta} \mathbb{1}_{\tau_{B_{R}(0)}(x, v)>\theta} d S(x) d v d \theta
\end{gathered}
$$

for any $t \geq 0$.

Proof. Let $p$ be the solution of the IBVP

$$
\frac{\partial p}{\partial t}+v \cdot \nabla_{x} p+a(t, x, v) p-K p=0, \quad(t, x, v) \in \mathbb{R}^{+} \times B_{R}(0) \times \mathbb{V}
$$

with initial and boundary conditions

$$
p(0, x, v)= \begin{cases}f^{\text {in }}(x, v) & x \in \Omega^{0} \times \mathbb{V} \\ 0 & x \in\left(B_{R}(0) \backslash \Omega^{0}\right) \times \mathbb{V},\left.\quad p(t, x, v)\right|_{(x, v) \in \Gamma^{-}}=0,\end{cases}
$$

where $\Gamma^{-}=\left\{(x, v) \in \partial B_{R}(0) \times \mathbb{V}: n_{x} \cdot v<0\right\}$, and $n_{x}$ is the outward normal to $B_{R}(0)$ originated in $x \in B_{R}(0)$.

Problem (4.1)-(4.2) can be written in the equivalent integral formulation

$$
\begin{gathered}
p(t, x, v)=f^{\text {in }}(x-t v, v) \exp \left(-\int_{0}^{t} a(s, x+(s-t) v, v) d s\right) \mathbb{1}_{\tau_{B_{R}(0)}(x, v)>t} \\
+\int_{\left(t-\tau_{B_{R}(0)}(x, v)\right)^{+}}^{t} \exp \left(-\int_{s}^{t} a(\tau, x+(\tau-t) v, v) d \tau\right) K p(s, x+(s-t) v, v) d s .
\end{gathered}
$$

Since the barrier $\Gamma^{t}$ remains confined in $B_{R}(0)$ for all $t \geq 0$, we have that

$$
\tau_{B_{R}(0)}(x, v) \geq \tau_{\Omega^{t}}(x, v)
$$

for all $(t, x, v) \in\left[0, t^{*}\right] \times \Omega^{t} \times \mathbb{V}$. The IBV problem (4.1)-(4.2) is posed in a fixed domain. It can hence be solved with the same strategy used in the proof of Theorem 3.1 and its solution can be written in terms of the Dyson-Phillips expansion. Its form is exactly as in Theorem 3.1, that is

$$
p=\sum_{n=0}^{+\infty} T_{B_{R}(0)}^{n} F_{B_{R}(0)}\left(f^{\text {in }}\right),
$$

where

$F_{B_{R}(0)}\left(f^{\mathrm{in}}\right)=f^{\mathrm{in}}(x-t v, v) \exp \left(-\int_{0}^{t} a(s, x+(s-t) v, v) d s\right) \mathbb{1}_{\tau_{B_{R}(0)}(x, v)>t}$ and

$T h=\int_{\left(t-\tau_{B_{R}(0)}(x, v)\right)^{+}}^{t} \exp \left(-\int_{s}^{t} a(\tau, x+(\tau-t) v, v) d \tau\right) K h(s, x+(s-t) v, v) d s$.

By comparison between the integral formulations (3.3) and (4.3) and the corresponding Dyson-Phillips expansions, we deduce immediately that

$$
0 \leq f \leq p \quad \text { for a.e. }(x, v) \in \Omega^{t} \times \mathbb{V}
$$

for all $t>0$. This implies that the exponential convergence to zero as $t \rightarrow+\infty$ for $f$ is a consequence of the same behaviour for $p$. 
Let us consider Equation (4.1). Since $p \geq 0$ for a.e. $(t, x, v) \in\left[0, t^{*}\right] \times$ $B_{R}(0) \times \mathbb{V}$ and $\bar{a} \geq\|k\|_{L^{\infty}\left(\left(0, t^{*}\right) \times B_{R}(0) \times \mathbb{V} \times \mathbb{V}\right)}$, we deduce that

$$
\begin{gathered}
\frac{d}{d t}\|p(t, \cdot, \cdot)\|_{L^{1}\left(B_{R}(0) \times \mathbb{V}\right)} \leq-\int_{B_{R}(0) \times \mathbb{V}} v \cdot \nabla_{x} p(t, x, v) d x d v \\
-\left(\bar{a}-\|k\|_{L^{\infty}\left(\left(0, t^{*}\right) \times B_{R}(0) \times \mathbb{V} \times \mathbb{V}\right)}\right)\|p(t, \cdot, \cdot)\|_{L^{1}\left(B_{R}(0) \times \mathbb{V}\right)}= \\
-\int_{\substack{\partial B_{R}(0) \times \mathbb{V} \\
v \cdot n_{x}>0}}\left|v \cdot n_{x}\right| p(t, x, v) d S(x) d v \\
-\left(\bar{a}-\|k\|_{L^{\infty}\left(\left(0, t^{*}\right) \times B_{R}(0) \times \mathbb{V} \times \mathbb{V}\right)}\right)\|p(t, \cdot, \cdot)\|_{L^{1}\left(B_{R}(0) \times \mathbb{V}\right)} \leq 0,
\end{gathered}
$$

where $d S(x)$ is the surface element on $\partial B_{R}(0)$, the last identity being a consequence of the divergence theorem.

By using the positivity of $p$ and of the kernel $k$, we obtain that

$$
p(t, x, v) \geq f^{\text {in }}(x-t v, v) e^{-t\|a\|_{L \infty\left(\mathbb{R}+\times B_{R}(0) \times \mathbb{V}\right)}} \mathbb{1}_{\tau_{B_{R}(0)}(x, v)>t}
$$

for a.e. $(x, v) \in B_{R}(0) \times \mathbb{V}$, for all $t>0$.

Hence, by Grönwall's lemma,

$$
\begin{aligned}
& \|p(t, \cdot, \cdot)\|_{L^{1}\left(B_{R}(0) \times \mathbb{V}\right)} \leq\left\|f^{\mathrm{in}}\right\|_{L^{1}\left(\Omega^{0} \times \mathbb{V}\right)} e^{-\left(\bar{a}-\|k\|_{L^{\infty}\left(\left(0, t^{*}\right) \times B_{R}(0) \times \mathbb{V} \times \mathbb{V}\right)}\right) t} \\
& -e^{-\left(\bar{a}-\|k\|_{L} \infty\right) t} \int_{0}^{t} \int_{\substack{\partial B_{R}(0) \times \mathbb{V} \\
v \cdot n_{x}>0}}\left|v \cdot n_{x}\right| f^{\text {in }}(x-\theta v, v) e^{-\|a\|_{L^{\infty}} \theta_{1}} \mathbb{1}_{\tau_{B_{R}(0)}(x, v)>\theta} d S(x) d v d \theta
\end{aligned}
$$

for any $t \geq 0$.

Hence, the result of the theorem follows.

The general case, with moving barriers that do not satisfy the prescriptions stated in Definition 2.1 and/or with cross sections inducing a local augmentation of the particle population, is much more complicated to study, and a wide variety of behaviours can happen.

In particular, it would be interesting to investigate whether the absorbing boundary can balance a net particle production inside the moving domain, in the regions where $k \geq a$.

Another interesting open question concerns the characterization of the requirements on $a, k$ and $\Gamma^{t}$ that lead to a finite extinction time of the density function $f$.

\section{The Numerical StRATEgy}

This sections is devoted to describe our numerical strategy for the study of (2.1)-(2.2). It is an extension of the particle method proposed in [8] and takes into account the effects of the medium on the particles, as described below.

In practice, a particle method simulates a population, composed by a great number of individual entities, by a reduced set of moving particles (whose number can be handled by a computing machine), which obey to the basic physical principles of the problem. For this reason, it allows the control of the main quantities used in the simulations, such as the mean free path between collisions, the particle free flow and the collision frequency. 
The strategy of a particle method consists in discretizing the unknown function $f$ by means of a sum of Dirac masses, centred in $\left(x_{k}(t), v_{k}(t)\right)_{1 \leq k \leq N}$, which represent a set composed by $N \in \mathbb{N}$ macro-particles that evolve in the phase space of a system. More precisely, the working hypothesis is the approximation

$$
f=\sum_{k=1}^{N} \omega_{k} \delta\left(x-x_{k}(t)\right) \delta\left(v-v_{k}(t)\right),
$$

where $\omega_{k}$ is the weight of the $k$-th particle.

Once the number $N$ of numerical particles has been chosen, the problem is initialized by approximating the initial condition $f^{\text {in }}$ with

$$
f^{\text {in }}(x, v)=\sum_{k=1}^{N} \omega_{k} \delta\left(x-x_{k}^{0}\right) \delta\left(v-v_{k}^{0}\right),
$$

and then the time evolution of the system is obtained by deducing the time evolution of the macro-particles on the characteristic curves of the problem (2.1)-(2.2).

Here, all the weights of the particles are identical, and are tuned in order to reproduce the mass of the initial condition:

for all $1 \leq k \leq N$.

$$
\omega_{k}=\left\|f^{\mathrm{in}}\right\|_{L^{1}\left(\Omega^{0} \times V\right)} / N,
$$

Different phenomena are responsible of the time evolution of the system. In particular, we need to take into account:

(1) the random walk of the particle in the domain, mathematically represented by the transport operator $v \cdot \nabla$ and by the collisional operator $(a-K)$;

(2) the absorbing conditions on the moving barrier;

(3) the time evolution of the absorbing barrier.

All these phenomena are taken into account in the code, by using the numerical strategy described below.

For simplicity, we will suppose always that the velocities of the macroparticles belong to the unit sphere, that is $v_{k}(t) \in \mathbb{S}^{d-1}$ for all $1 \leq k \leq N$ and for all $t \geq 0$, and that the system is locally conservative and spatially homogeneous, i.e.

$$
a(t, x, v)=\sigma>0 \text { and } k(t, x, v, w)=\sigma /\left|\mathbb{S}^{d-1}\right|>0
$$

for a.e. $(t, x, v, w) \in \mathbb{R}^{+} \times \mathbb{R}^{d} \times \mathbb{S}^{d-1} \times \mathbb{S}^{d-1}$.

5.1. Motion of the particle with "frozen" absorbing barrier. We choose a fixed small time interval $\Delta t>0$, whose magnitude is smaller (of at least one order of magnitude) than the time corresponding to the mean free path of the particle gas and fix the fraction $\alpha \in(0,1)$ of particles that are supposed to change direction through scattering phenomena in $[t, t+\Delta t]$.

Then, in the time interval $[t, t+\Delta t]$ the numerical particles move according to the free flow rule

$$
\left\{\begin{array}{l}
x_{k}^{\prime}(t)=v_{k}(t), \\
v_{k}^{\prime}(t)=0
\end{array}\right.
$$


under the initial conditions $\left(x_{k}(0), v_{k}(0)\right)=\left(x_{k}^{0}, v_{k}^{0}\right)$, for all $1 \leq k \leq N$.

The next step consists in generating a random number from an uniform distribution between the interval $(0,1)$. If this value is less or equal to $\alpha$, the particles is considered to be scattered by the medium, in the opposite case the particles will be driven by the free flow with velocity $v_{k}(t)$. Note that $\alpha$ depends on the time step $\Delta t$ that has been chosen in the numerical simulations: if $\alpha \ll 1$ for a particular choice of $\Delta t$, then there exists a simple rule for computing the fraction of scattered particles $\alpha_{*}>0$ for a multiple $\Delta t_{*}=m \Delta t, m \in \mathbb{N}$ : indeed, the probability that a particle follows a free flow dynamics in the time interval $\left[t, t+\Delta t_{*}\right]=[t, t+m \Delta t]$ can be written either as $(1-\alpha)^{m}$ or $\left(1-\alpha_{*}\right)$ respectively.

But, when $\alpha \ll 1$, we have that $(1-\alpha)^{m} \approx(1-m \alpha)$, and hence $\alpha_{*} \approx m \alpha$.

The link between $\alpha$ and $\sigma$ is then given by the relationship

$$
\sigma=\alpha / \Delta t .
$$

In particular, this procedure generates a sequence of changes in the velocity directions whose time intervals $\left(\theta_{n}\right)_{n \geq 0}$ in $\mathbb{R}^{+}$are described, in the limit $\Delta t \rightarrow 0$, by an exponential law of parameter $\sigma$ : for $t>0$,

$$
\mathbb{P}\left(\theta_{n}>t\right)=e^{-\sigma t} \text {. }
$$

For $n \geq 1$, the total time after $n$ collisions is obviously

$$
T_{n}=\sum_{j=0}^{n-1} \theta_{j} .
$$

On the other hand, the effect of the scattering is obtained through a sample of the velocity $v_{k}$ on the sphere $\mathbb{S}^{d-1}$, with an uniform probability law. By denoting with $\left(V_{n}\right)_{n \geq 1}$ a sequence of independent random variables in $\mathbb{S}^{d-1}$ with uniform law, representing the velocity changes, we have that

$$
\mathbb{P}\left(V_{n} \in V \subseteq \mathbb{S}^{d-1}\right)=\frac{1}{\left|\mathbb{S}^{d-1}\right|} \int_{V} d \omega .
$$

It is well known that the procedure written above generates a density function $f$ that solves the transport equation $(2.1)$, with $V=\mathbb{S}^{d-1}, a(t, x, v)=$ $\sigma>0$ for a.e. $(t, x, v) \in \mathbb{R}^{+} \times \Omega^{t} \times V$ and with $k(t, x, v, w)=\sigma /\left|\mathbb{S}^{d-1}\right|$ for a.e. $(t, x, v, w) \in \mathbb{R}^{+} \times \Omega^{t} \times V \times V$ (see, for example, [1]).

This procedure is performed by supposing that the absorbing boundary is fixed in its position at time $t$ for the whole duration of the time step, i.e. the random walk takes places in $\Omega^{t}$.

5.2. Time evolution of the absorbing barrier. The second step consists in taking into account the time evolution of the absorbing surface $\Gamma^{t}$ in the time interval $[t, t+\Delta t]$, the time step $\Delta t$ being the same as in Subsection 5.1 .

The motion of the barrier is treated in an exact way, provided that an analytical formulation of its time evolution is known.

Then, the algorithm identifies between the numerical particles, described by the sum

$$
f=\sum_{k=1}^{N} \omega_{k} \delta\left(x-x_{k}(t+\Delta t)\right) \delta\left(v-v_{k}(t+\Delta t)\right),
$$


those that leave the domain in the time interval $[t, t+\Delta t]$. In this case, the corresponding weights $\omega_{k}$ are set to zero and then the corresponding particle is removed.

If the final time has not been reached, then the two steps of the splitting procedure are performed again in the next time interval $[t+\Delta t, t+2 \Delta t]$, with initial conditions $\left(x_{k}(t+\Delta t), v_{k}(t+\Delta t)\right)$, for all $1 \leq k \leq N^{*}$, where $N^{*} \leq N$ represents the new total number of particles, obtained by subtracting from $N$ the number of particles absorbed by the moving barrier.

5.3. Advantages and drawbacks of the numerical strategy. As all numerical approaches to a partial differential equation, the particle method presented here has some weaknesses and some positive aspects.

In primis, it is very simple to implement, even for complex geometries of the evolutionary barrier and in situations of high dimensionality. The evolution law of the barrier is treated in an exact way, provided that its analytical formulation is known.

Our strategy is based on the physical hypotheses that allow to deduce the linear Boltzmann equation itself, starting from an Hamiltonian system of point particles. However, the number of particles that is needed in order to achieve a reasonable accuracy is very large.

In order to reduce the computation time, a parallelization technique (for example, on graphic cards) could be used.

We refer to [8] for details concerning the parallelization of a particle method in the case of a Knudsen gas. This strategy could be easily adapted to the present case.

\section{Numerical RESUlts}

In this section we show some relevant examples of long-time behaviour in different situations.

All computations have been performed in the three-dimensional physical space $\mathbb{R}^{3}$, without taking into account any symmetry, and with velocities in $\mathbb{S}^{2}$. Hence, the simulation took place in the five-dimensional phase-space $\mathbb{R}^{3} \times \mathbb{S}^{2}$.

In all tests we used $N=10^{6}$ numerical particles, and a time step $\Delta t=$ $2 \times 10^{-4}$. The initial condition of all simulations is a weighted characteristic function of the ball of radius $1 / 2$ centred in $(-.5,0,0) \in \mathbb{R}^{3}$, with a normalization factor such that $\left\|f^{\text {in }}\right\|_{L^{1}\left(\mathbb{R}^{3} \times \mathbb{S}^{2}\right)}=1$ :

$$
f^{\text {in }}(x, v)=\frac{6}{\pi} \mathbb{1}_{(x, v) \in B_{1 / 2}((-.5,0,0)) \times \mathbb{S}^{2} .}
$$

The time evolution of $f^{\text {in }}$ is given by the problem

$$
\begin{gathered}
\frac{\partial f}{\partial t}+v \cdot \nabla_{x} f=\sigma\left(\int_{\mathbb{S}^{2}} f(t, x, w) d w-f\right)=0, \quad(t, x, v) \in \mathbb{R}^{+} \times \Omega^{t} \times \mathbb{S}^{2}, \\
(6.2) \quad f(0, x, v)=f^{\text {in }}(x, v),\left.\quad f(t, x, v)\right|_{x \in \Gamma_{i}^{t}}=0,
\end{gathered}
$$

where $\Gamma_{i}^{t}(i=1, \ldots, 4)$ are some relevant absorbing moving barriers. In what follows, we denote with $x=\left(x_{1}, x_{2}, x_{3}\right) \in \mathbb{R}^{3}$. In all tests, $\sigma=10$. 

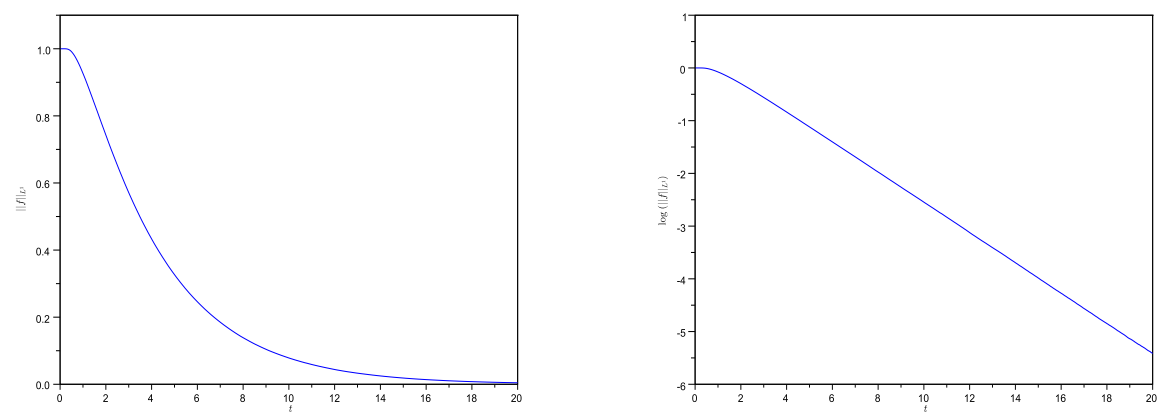

Figure 1. Time evolution of the solution of (6.1)-(6.2) with absorbing barrier $\mathbb{S}^{2}:\|f\|_{L^{1}}$ versus $t$ (left) and $\log \left(\|f\|_{L^{1}}\right)$ versus $t$ (right).

The simulations, written in C, heavily need sequences of random numbers. In our tests, we used the standard rand48() pseudo-random number generator, which guarantees a satisfactory approximation of the uniform distribution.

The hardware being used is a workstation equipped with an Intel Xeon CPU X5450 (12M Cache, $3.00 \mathrm{GHz}, 1333 \mathrm{MHz}$ FSB), with 47 GB memory.

6.1. First example: fixed domain. We consider (6.1)-(6.2) under the effect of the fixed absorbing barrier $\Gamma_{1}^{t}=\mathbb{S}^{2}$, i.e. the barrier is the unit sphere.

In Figure 1, we show that the $L^{1}$-norm of $f$ is exponentially decaying to zero after a transient initial period that corresponds to the time interval that is needed to the first particles for reaching the absorbing boundary.

6.2. Second example: expansion. We now consider the problem defined by (6.1)-(6.2) under the action of the absorbing moving barrier

$$
\Gamma_{2}^{t}=\left\{x \in \mathbb{R}^{3}: x_{1}^{2}+x_{2}^{2}+x_{3}^{2}=e^{-t}+3\left(1-e^{-t}\right), t \in \mathbb{R}^{+}\right\} .
$$

$\Gamma_{2}^{t}$ is a sphere of radius $r=1$ at time $t=0$, that expands in time and whose radius reaches asymptotically the value $r=3$ as $t \rightarrow+\infty$.

The results, plotted in Figure 2, show that the $L^{1}$-norm of $f$ is strictly decreasing in time, after a transient initial period corresponding to the smallest forward exit time of the particles that describe the initial condition.

Here the convergence speed is slower with respect to the first simulation. This result agrees with the physics of the problem, since the expansion of the absorbing boundary induces a modification of the forward absorbing time, which is larger than the forward absorbing time obtained in Subsection 6.1:

$$
\tau_{\Omega_{2}^{t}}(x, v) \geq \tau_{\Omega_{1}^{t}}(x, v) \text { for all }(t, x, v) \in \mathbb{R}^{+} \times\left(\Omega_{1}^{t} \cap \Omega_{2}^{t}\right) \times \mathbb{S}^{2} .
$$

Also in this case the convergence is exponential for large time. 

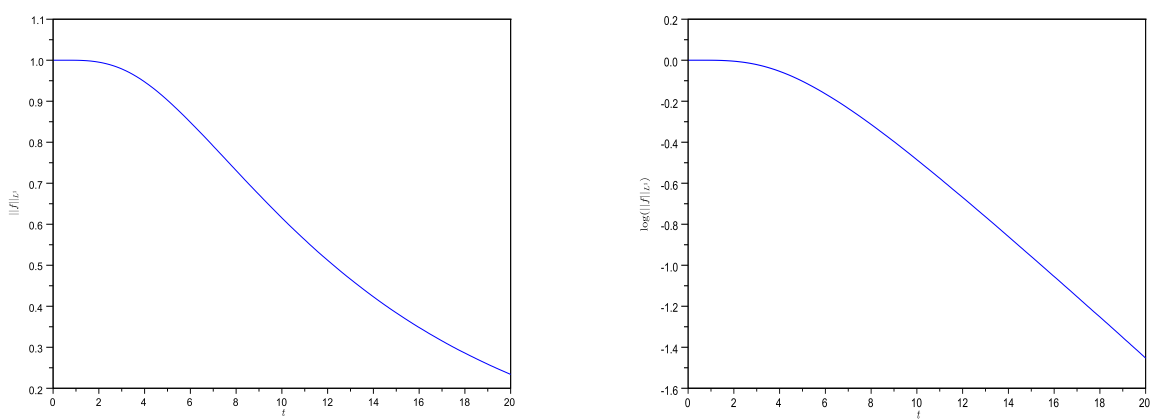

Figure 2. Time evolution of the solution of (6.1)-(6.2) under the action of $\Gamma_{2}^{t}$ : $\|f\|_{L^{1}}$ versus $t$ (left) and $\log \left(\|f\|_{L^{1}}\right)$ versus $t$ (right).
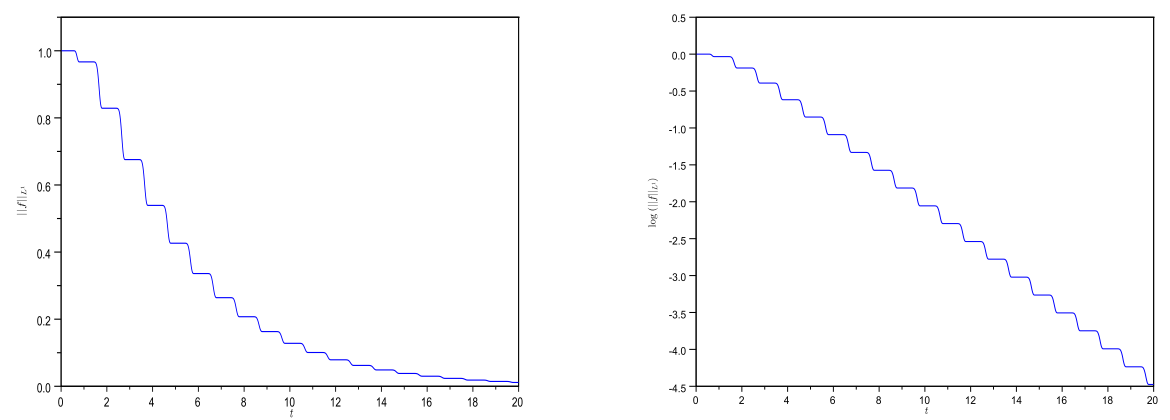

Figure 3. Time evolution of the solution of (6.1)-(6.2) with absorbing barrier $\Gamma_{3}^{t}$ : $\|f\|_{L^{1}}$ versus $t$ (left) and $\log \left(\|f\|_{L^{1}}\right)$ versus $t$ (right).

6.3. Third example: oscillatory domain. The next simulation shows the behaviour of the solution of Problem (6.1)-(6.2), under the action of the oscillatory moving barrier

$$
\Gamma_{3}^{t}=\left\{x \in \mathbb{R}^{3}: x_{1}^{2}+x_{2}^{2}+x_{3}^{2}=2+\sin (2 \pi t), t \in \mathbb{R}^{+}\right\} .
$$

The results, plotted in Figure 3 (left), show that the $L^{1}$-norm of $f$ is globally monotone non increasing.

However, during the expansion phases of the barrier, sometimes the velocity of the particles is smaller than the expansion velocity of the moving boundary. In this case, no particles can reach the absorbing barrier and therefore the $L^{1}$-norm of $f$ remains constant on such time subintervals. As shown by the semi-logarithmic plot (Figure 3, right), it is apparent that the convergence is exponential for large time.

6.4. Fourth example: translational barrier. In this last simulation, we consider a surface whose evolution law includes translational effects. In 


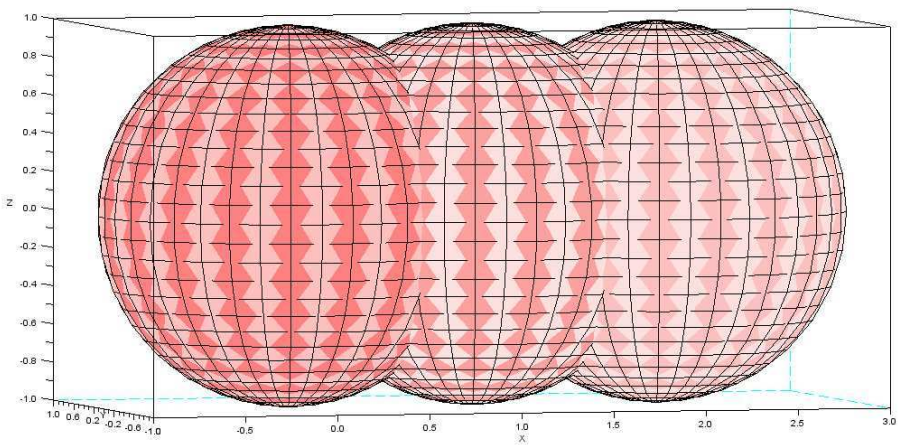

Figure 4. Time evolution of the absorbing barrier $\Gamma_{4}^{t}$ at $t=0$ (left), $t=0.5$ (centre) and $t=1$ (right).
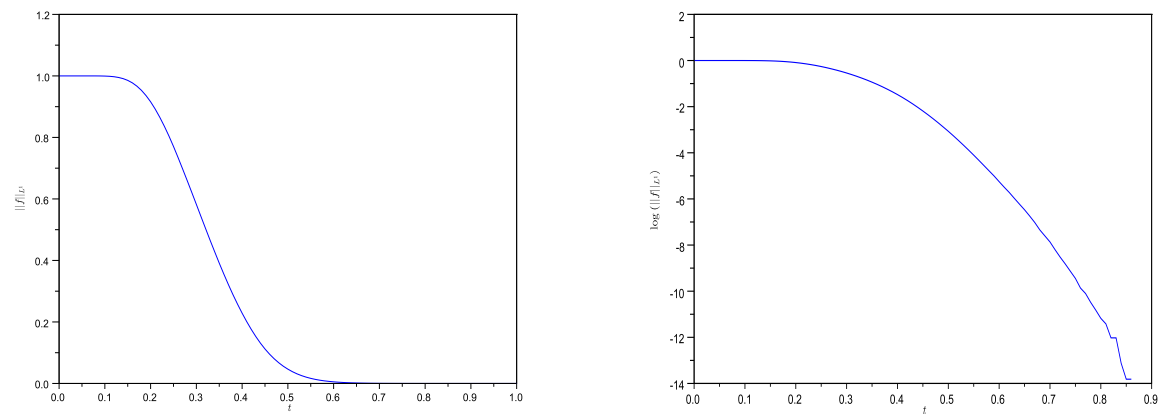

Figure 5. Time evolution of the solution of (6.1)-(6.2) with absorbing barrier $\Gamma_{4}^{t}$ : $\|f\|_{L^{1}}$ versus $t$ (left) and $\log \left(\|f\|_{L^{1}}\right)$ versus $t$ (right).

particular, Problem (6.1)-(6.2) is set under the effect of the moving boundary

$$
\Gamma_{4}^{t}=\left\{x \in \mathbb{R}^{3}:\left(x_{1}-2 \varphi(t)\right)^{2}+x_{2}^{2}+x_{3}^{2}=1, t \in \mathbb{R}^{+}\right\},
$$

where $\varphi(t)=t$ for $t \in[0,1]$ and $\varphi(t)=1$ for $t \geq 1$ (see Figure 4 for a visualization of the time evolution of the absorbing barrier).

This simulation shows that, sometimes, the convergence to the long-time equilibrium can be better than exponential. In particular, in Figure 5, we numerically recover a convergence to zero in finite time. This behaviour is due to the fact that there exists a time $\tilde{t}$ for which the support of $f$ and $\Omega_{4}^{t}$ are disjoint.

The slight degradation in the time interval $[0.75,0.9]$ of the numerical simulation is caused by the reduced number of numerical particles: at time $t=0.7500$ only 79 numerical particles are present, and the last one is absorbed by the moving boundary at time $t=0.8646$. 


\section{Conclusions}

We have studied the behaviour of the linear Boltzmann equation under the action of a moving barrier in $\mathbb{R}^{d}, d \in \mathbb{N}$. Existence and uniqueness of the solution have been proved, as well as the exponential asymptotic convergence to zero as $t \rightarrow+\infty$ in the absorption dominated case.

We have then proposed a numerical strategy for describing our problem, based on a particle method.

The results of this article can be extended in many aspects. From a theoretical point of view, it would be interesting to investigate on a more detailed basis the relationships between the initial data and the evolution law of the moving barrier in order to deduce more precise convergence results in some specific regimes. Furthermore, it would be interesting to take into account more complex interactions with the boundary, and explore, both theoretically and numerically, the resulting kinetic models and their fluid limits.

Acknowledgements. The idea for this article was originated during the programme "Partial Differential Equations in Kinetic Theories" at the Isaac Newton Institute (Cambridge, UK), which is kindly acknowledged for its hospitality. This paper has been partially supported by the Italian national institute of higher mathematics (INDAM), GNFM project "Study of complex kinetic systems: theoretical analysis and numerical simulation". The author thanks Ludovico Pernazza for useful discussions on the subject of the moving barriers.

\section{REFERENCES}

[1] Grégoire Allaire and François Golse. Trasport et diffusion. École Polytechnique, Palaiseau, 2012.

[2] Kazuo Aoki, Guido Cavallaro, Carlo Marchioro, and Mario Pulvirenti. On the motion of a body in thermal equilibrium immersed in a perfect gas. M2AN Math. Model. Numer. Anal., 42(2):263-275, 2008.

[3] Étienne Bernard and Francesco Salvarani. Optimal estimate of the spectral gap for the degenerate goldstein-taylor model, 2012.

[4] Étienne Bernard and Francesco Salvarani. On the Convergence to Equilibrium for Degenerate Transport Problems. Arch. Ration. Mech. Anal., 208(3):977-984, 2013.

[5] Étienne Bernard and Francesco Salvarani. On the exponential decay to equilibrium of the degenerate linear boltzmann equation. J. Funct. Anal., (in press), 2013.

[6] Kenneth M. Case and Paul F. Zweifel. Linear transport theory. Addison-Wesley Publishing Co., Reading, Mass.-London-Don Mills, Ont., 1967.

[7] S. Chandrasekhar. Radiative Transfer. Oxford University Press, 1950.

[8] Florian De Vuyst and Francesco Salvarani. Gpu-accelerated numerical simulations of the knudsen gas on time-dependent domains. Comput. Phys. Comm., 184(3):532-536, 2013.

[9] Florian De Vuyst and Francesco Salvarani. Numerical simulations of degenerate transport problems, 2013.

[10] Laurent Desvillettes and Francesco Salvarani. Asymptotic behavior of degenerate linear transport equations. Bull. Sci. Math., 133(8):848-858, 2009.

[11] Luca Formaggia, Alfio Quarteroni, and Alessandro Veneziani, editors. Cardiovascular mathematics, volume 1 of MSEA. Modeling, Simulation and Applications. SpringerVerlag Italia, Milan, 2009. Modeling and simulation of the circulatory system.

[12] Dimitri Mihalas and Barbara Weibel Mihalas. Foundations of radiation hydrodynamics. Oxford University Press, New York, 1984. 
[13] Tetsuro Tsuji and Kazuo Aoki. Decay of a linear pendulum in a free-molecular gas and in a special Lorentz gas. J. Stat. Phys., 146(3):620-645, 2012.

Dipartimento di Matematica F. Casorati, Università degli Studi di Pavia, Via Ferrata 1, I-27100 PAVia, Italy

E-mail address: francesco.salvarani@unipv.it 